\title{
Clinicopathological value of long non-coding RNA profiles in gastrointestinal stromal tumor
}

\author{
Yan Zhao ${ }^{\text {Equal first author, } 1}$, Xinxin Liu ${ }^{\text {Corresp., Equal first author, } 2}{ }^{2}$, Keshuai Xiao ${ }^{3}$, Liwen Wang $^{2}$, Yuping $^{2}{ }^{2}{ }^{2}$ Mingyun Kan $^{2}$, Zhiwei $^{2}$ \\ Jiang ${ }^{\text {Corresp. } 2}$ \\ ${ }^{1}$ Clinical Medical College of Yangzhou University, Northern Jiangsu People's Hospital, Yangzhou, Jiangsu Province, China \\ Department of Gastrointestinal Surgery, Jiangsu Province Hospital of Traditional Chinese Medicine, Nanjing, Jiangsu Province, China \\ 3 Department of General Surgery, Yangzhou Hongquan Hospital, Yangzhou, Jiangsu Province, China \\ Corresponding Authors: Xinxin Liu, Zhiwei jiang \\ Email address: gorilla1999@hotmail.com, surgery34@163.com
}

Background. Long non-coding RNAs (IncRNAs) have been implicated in diagnosis and prognosis in various cancers. However, few IncRNA signatures have been established for prediction of gastrointestinal stromal tumors (GIST). We aimed to explore a IncRNA signature profile that associated with clinical relevance by mining data from Gene Expression Ominus (GEO) and Surveillance, Epidemiology, and End Results (SEER) Program.

Methods. Using a IncRNA-mining approach, we performed non-negative matrix factorization (NMF) consensus algorithm in Gastrointestinal stromal tumors (GISTs) cohorts (61 patients from GSE8167 and GSE17743) to cluster LncRNA expression profiles. Comparative markers selection, and Gene Set Enrichment Analysis (GSEA) algorithm were performed between distinct molecular subtypes of GIST. The survival rate of GIST patients from SEER stratified by gender were compared by Kaplan-Meier method and log-rank analysis. IncRNA-mRNA co-expression analysis was performed by Pearson correlation coefficients (PCC) using R package LINC. Somatic copy number alterations of GIST patients (GSE40966) were analyzed via web server GenePattern GISTIC2 algorithm.

Results. Four IncRNA molecular subtypes of GIST were identified with distinct biological pathways and clinical characteristics. LncRNA expression profiles well clustered the GIST samples into small size $(<5 \mathrm{~mm})$ and large size tumors $(>5 \mathrm{~mm})$, which is a fundamental index for GIST malignancy diagnosis. Several IncRNAs with abundant expression (LRRC75A-AS1, HYMAI, NEAT1, XIST and FTX) were closely associated with tumor size, which may suggest to be biomarkers for the GIST malignancy. Particularly, LRRC75A-AS1 was positively associated with tumor diameters and suggested an oncogene in GIST. Coexpression analysis suggested that chromosome region 17p11.2-p12 may contribute to the oncogenic process in malignant GIST. Interestingly, the gender had a strong influence on clustering by IncRNA expression profile. Data from Surveillance, Epidemiology, and End Results (SEER) Program were further explored and 7983 patients who were diagnosed with GISTs from 1973 to 2014 were enrolled for analysis. The results also showed the favorable prognosis for female patients. The survival rate between male and female with GIST was statistically significant $(P<0.0001)$. Gene set enrichment analysis (GSEA) indicated distinct pathways between female and male, and malignant GIST was associated with several cancer metabolism and cell cycle associated pathways.

Conclusions. This IncRNAs-based classification for GISTs may provide a molecular classification applicable to individual GIST that has implications to influence IncRNA markers selection and prediction of tumor progression. 
2 Clinicopathological value of long non-coding RNA profiles in gastrointestinal

4

5 Yan Zhao Equal first author, 1, Xinxin Liu Corresp., Equal first author, 2, Keshuai Xiao ${ }^{3}$, Liwen Wang ${ }^{2}$, Yuping $6 \mathrm{Li}^{2}$, Mingyun Kan ${ }^{2}$, Zhiwei Jiang Corresp, 2

7

8

${ }^{1}$ Clinical Medical College of Yangzhou University, Northern Jiangsu People's Hospital, Yangzhou, Jiangsu Province, China

${ }^{2}$ Department of Gastrointestinal Surgery, Jiangsu Province Hospital of Traditional Chinese

Corresponding Authors: Medicine, Nanjing, Jiangsu Province, China

${ }^{3}$ Department of General Surgery, Yangzhou Hongquan Hospital, Yangzhou, Jiangsu Province, China

Email address: gorilla1999@hotmail.com; surgery34@163.com

\section{Abstract}

Background. Long non-coding RNAs (lncRNAs) have been implicated in diagnosis and prognosis in various cancers. However, few lncRNA signatures have been established for prediction of gastrointestinal stromal tumors (GIST). We aimed to explore a lncRNA signature profile that associated with clinical relevance by mining data from Gene Expression Ominus (GEO) and Surveillance, Epidemiology, and End Results (SEER) Program.

Methods. Using a lncRNA-mining approach, we performed non-negative matrix factorization (NMF) consensus algorithm in Gastrointestinal stromal tumors (GISTs) cohorts (61 patients from GSE8167 and GSE17743) to cluster LncRNA expression profiles. Comparative markers selection, and Gene Set Enrichment Analysis (GSEA) algorithm were performed between distinct molecular subtypes of GIST. The survival rate of GIST patients from SEER stratified by gender were compared by Kaplan-Meier method and log-rank analysis. lncRNA-mRNA coexpression analysis was performed by Pearson correlation coefficients (PCC) using R package LINC. Somatic copy number alterations of GIST patients (GSE20709) were analyzed via web server GenePattern GISTIC2 algorithm. 
36 Results. Four lncRNA molecular subtypes of GIST were identified with distinct biological

37 pathways and clinical characteristics. LncRNA expression profiles well clustered the GIST

38 samples into small size $(<5 \mathrm{~mm})$ and large size tumors $(>5 \mathrm{~mm})$, which is a fundamental index for

39 GIST malignancy diagnosis. Several lncRNAs with abundant expression (LRRC75A-AS1,

40 HYMAI, NEAT1, XIST and FTX) were closely associated with tumor size, which may suggest

41 to be biomarkers for the GIST malignancy. Particularly, LRRC75A-AS1 was positively

42 associated with tumor diameters and suggested an oncogene in GIST. Co-expression analysis

43 suggested that chromosome region 17p11.2-p12 may contribute to the oncogenic process in

44 malignant GIST. Interestingly, the gender had a strong influence on clustering by lncRNA

45 expression profile. Data from Surveillance, Epidemiology, and End Results (SEER) Program

46 were further explored and 7983 patients who were diagnosed with GISTs from 1973 to 2014

47 were enrolled for analysis. The results also showed the favorable prognosis for female patients.

48 The survival rate between male and female with GIST was statistically significant $(\mathrm{P}<0.0001)$.

49 Gene set enrichment analysis (GSEA) indicated distinct pathways between female and male, and

50 malignant GIST was associated with several cancer metabolism and cell cycle associated

51 pathways.

52 Conclusions. This lncRNAs-based classification for GISTs may provide a molecular

53 classification applicable to individual GIST that has implications to influence lncRNA markers

54 selection and prediction of tumor progression.

55

56 Introduction

57 Gastrointestinal stromal tumors (GISTs) constitute the most common form of subepithelial tumor

58 of the gastrointestinal tract (Xu et al. 2014; Yan et al. 2015). Approximately $60 \%$ to $70 \%$ of

59 GISTs arise in the stomach, $20 \%$ to $30 \%$ in the small intestine, and $5 \%$ in the colon and rectum

60 (Corless et al. 2004; Miettinen \& Lasota 2001). On the basis of similarities in

61 immunohistochemical and ultrastructural features, it is considered that GISTs arise from

62 interstitial cells of Cajal (ICC) or their precursor cells (Kindblom et al. 1998). More than 80\% of

63 GISTs have gain of function mutations of the KIT proto-oncogene that encodes the c-Kit

64 (CD117) (Hirota et al. 1998), and the PDGFRA gene that encodes platelet-derived growth factor

65 receptor (PDGFRA) tyrosine kinase (Hirota et al. 2003). Nevertheless, GISTs present extremely

66 heterogeneous clinical prognosis. Currently, the criteria for risk estimation depend largely on 
67 clinicopathologic factors, such as tumor site, size, cell type, degree of necrosis, mitotic rate, Ki-

68

69

70

71

72

73

74

75

76

77

78

79

80

81

82

83

84

85

86

87

88

89

90

91

92

93

94

95

96

97

98

67 immunoreactivity as well as their combinations (DeMatteo et al. 2000; Miettinen et al. 2002).

Increasing evidence suggested that the aberrant expression of long non-coding RNAs (lncRNAs) have been associated with cancer initiation and progression (Mitra et al. 2012; Yoon et al. 2015), and some of them have been implicated in diagnosis and prognosis (Qi \& Du 2013). Moreover, lncRNAs emerge as strong cancer biomarkers as it has higher specificity than proteincoding mRNAs (Hessels et al. 2003; Prensner \& Chinnaiyan 2011), and stably detectable in the blood (Lin et al. 2007) and urine (Hessels et al. 2003; Tinzl et al. 2004) by conventional RT-PCR methods. In GIST, studies have shown that lncRNA HOTAIR could be one of the best candidates as potential prognostic biomarkers (Lee et al. 2016; Niinuma et al. 2012). FFPE tissue specimens data from 40 surgically resected and metastatic GIST patients suggest a potential biomarker and prognostic value of both H19 and MALAT1 lncRNAs for the clinical selection of the best candidate to first-line treatment with imatinib (Badalamenti et al. 2019). LncRNA AOC4P in 79 GIST patients' tissues in high risk GIST tissues was higher than that in low/medium-risk GIST tissues and normal tissues(Hu et al. 2018). In vitro cell culture study showed lncRNA AOC4P silencing can reduce the proliferative ability, decrease the migration and invasion activity, and induce cell apoptosis(Hu et al. 2018). Studies by using chip technology also identified a panel of dysregulated lncRNAs that may serve as potential biomarkers or drug targets for GISTs, particularly secondary imatinib-resistant GISTs(Yan et al. 2019). Therefore, searching a lncRNA signature might suggest a potential biomarker and prognostic value for GIST.

LncRNA profiling analysis could be realized by mining previously published gene expression microarray due to a large group of lncRNA-specific probes present on the commonly used microarray platforms. Multiple studies have discovered new lncRNA biomarkers and identified therapeutic lncRNA targets using mining approach of re-annotating microarray (Zhang et al. 2012; Zhu et al. 2016). In this study, we analyzed a well-characterized cohort of GIST microarrays from the Gene Expression Omnibus (GEO) in order to clarify the gene expression or lncRNA alterations associated with clinicopathological value. The Surveillance, Epidemiology, and End Results (SEER) Program of the US National Cancer Institute (NCI) was also used to identify GIST patients' overall survival and investigate molecular features that might be applicable to the prediction of outcome. 


\section{Materials \& Methods}

\section{GIST gene expression datasets preparation}

101 Microarray data of GSE20710_RAW (Astolfi et al. 2010), GSE56670_RAW (Killian et al. 102 2014), GSE8167_RAW (Yamaguchi et al. 2008) and GSE17743_RAW (Ostrowski et al. 2009)

103 were directly downloaded from Gene Expression Omnibus databases. These datasets

104 corresponded to all available public datasets fulfilling the following criteria: available gene 105 expression data obtained using the same chip platform (Affymetrix U133 Plus 2.0 chips) with 106 raw data CEL files, and some associated clinicopathological data were available. SDH-deficient 107 gastrointestinal stromal tumors (Killian et al. 2014) were excluded, because of the small 108 percentage of GIST and totally different gene-expression style (Janeway et al. 2011; Miettinen et

109

110

111

112

113

114

115

116

117

118

119

120

121

122

123

124

125

126

127

128

129

al. 2011). Gene expression's background correction, normalization and summarizing were performed using Robust Multichip Average (RMA) (Irizarry et al. 2003b) by TCA software (Affymetrix Co., Santa Clara, CA, US) (Irizarry et al. 2003a).

\section{Microarray data processing and IncRNA profile mining}

The approach of lncRNA profile mining mainly referred to Haoyan Chen et al (Hu et al. 2014; Zhu et al. 2016) and Xiaoqin Zhang et al (Zhang et al. 2012). Briefly, the Affymetrix HG-U133 Plus 2.0 probe set IDs were mapped to the NetAffx Annotation Files and gene biotypes were further validated by BioMart databases (biomaRt R package). Based on the Refseq transcript ID and/or Ensembl gene ID, we only retained non-coding protein genes and further filtered them by eliminating pseudogenes including microRNAs, rRNAs and other short RNAs such as snoRNAs, snRNAs and tRNAs. Finally, 2448 annotated lncRNA transcripts with corresponding Affymetrix probe IDs were generated. Some lncRNA signatures were well confirmed by previous study (Hu et al. 2014; Zhu et al. 2016).

\section{LncRNA markers selection and expression style between subtypes}

The subtypes of GIST were identified in row median centered microarray datasets using the nonnegative matrix factorization (NMF) algorithm (Brunet et al. 2004). We used the Comparative Marker Selection algorithm (Gould et al. 2006) by GenePattern (https://genepattern.broadinstitute.org/gp). One-versus-all comparisons were performed to identify differentially expressed genes that can discriminate between distinct lncRNA clustering 
130

131

132

133

134

135

136

137

138

139

140

141

142

143

144

145

146

147

148

149

150

151

152

153

154

155

156

157

158

159

160

161

subtypes. Strict criteria were used to select the lncRNA markers, including fold change of gene expression $>2$, FDR $(\mathrm{BH})<0.05$, Feature-Specific $\mathrm{p}$-Value $<0.05$.

\section{Identification of biological pathways distinguishing each subtype}

We performed GSEA through the JAVA program (http://www.broadinstitute.org/gsea) by using MSigDB C2 CP: Canonical pathways gene set collection (1320 gene sets available), and visualized the GSEA outputs in Cytoscape (version 3.4). Then we used the Enrichment Map software to identify the biological processes distinguishing one subtype from others. In order to simplify the network map, we chose a strict threshold of gene-set permutations with an FDR cutoff of 5\% and p-value cutoff of $1 \%$ in Enrichment Map software.

\section{SEER database exploration}

SEER data was used to identify patients that were diagnosed as GISTs from 1973 to 2014 (Cronin et al. 2014). The exclusion criterions included: age $<18$, no evaluation of histological type, not the first diagnosed malignancy, an unknown cause of death or survival month. The primary outcomes of interest were overall survival (OS), which was calculated from the date of diagnosis to the date of attributed death.

To better explore gender influence on the survival rate, the propensity-score-matched analyses were performed with equal factors which may influence the prognosis. Pairing criteria included tumor sizes, grade, age, treatment. Survival function estimation was performed with the Kaplan-Meier method and the resulting curves compared with the log-rank test. All statistical analyses were computed using SPSS version 24 (IBM Corporation, Armonk, NY, USA).

\section{IncRNA-mRNA co-expression network construction and gene annotation}

R package LINC provided methods to compute co-expression networks of lincRNAs and protein-coding genes. Biological terms associated with the sets of protein-coding genes predicted the biological contexts of lincRNAs according to the 'Guilty by Association' approach (Gillis \& Pavlidis 2012). This R package LINC applied this idea on arbitrary expression matrices (Yu et al. 2015) and could reveal which functions, pathways or compartments were associated with the lncRNA co-expressed genes.

\section{Somatic copy number alterations (CNA) frequency profiles}


162 We downloaded CNA array data of patients via the NCBI Gene Expression Omnibus (accession

163 number GSE20709), and analyzed the raw copy number data for each GIST sample provided by

164 array comparative genomic hybridization $(\mathrm{CGH})$ by using the Copy Number Inference Pipeline

165 for SNP6 CEL files and GISTIC2 algorithm (Mermel et al. 2011) via web server GenePattern.

166

167 Results

168 Classification of GISTs into four subtypes based on extracted IncRNA profiles

169 Due to the limited chipset data available for GIST, only two datasets GSE8167_RAW

170 (Yamaguchi et al. 2008) and GSE17743_RAW (Ostrowski et al. 2009) were adapted as main

171 data source in this study. The clinicopathological, immunohistochemical, and genetic

172 characteristics of the 61 cases of GIST used in the chipset analysis are summarized in

173 supplementary table 1.

174 To explore the overall gene expression pattern, we first performed unsupervised analysis of 175 all 54,613 probe sets and with 20647 collapsed genes. Consensus NMF clustering (Brunet et al.

176 2004) separated the 61 GISTs into three principal classes ( $\max$ coef. $=0.9929$, when $\mathrm{k}=3$ ). To

177 eliminate probes that had little or no variation across samples, we then keep the $50 \%$ most

178 varying probes to run the non-negative matrix factorization (NMF) algorithm again. Next, we

179 repeated the same unsupervised analysis with extracted lncRNA probe sets. According to the

180 cophenetic coefficient scores from the consensus NMF clustering ( $k=2$ to $k=6)$, we chose to

181 cluster 61 GISTs into four subtypes (max coef.=0.9932, when $\mathrm{k}=4)$ (Fig. 1A-F). The four

182 clustering subtypes were designated as G1, G2, G3, and G4. Metagenes analysis and linkage tree

183 of extracted lncRNAs ordered by $\mathrm{k}=4$ clustering subtypes were showed in Figure $1 \mathrm{G}-\mathrm{I}$. Thus, as

184 previously reported (Brunet et al. 2004), NMF with consensus clustering and hierarchical

185 dendrogram gave strong evidence for four classes split of the lncRNAs with a correspondingly

186 high cophenetic coefficient.

187

188 LncRNAs clustering GIST subtypes were associated with tumor size and gender.

189 Subtypes clustered by global gene expression ( $\max$ coef. $=0.9929, \mathrm{k}=3$ ) did not show any 190 meaning to the clinicopathological value, as well as extracted most varying probes. However, 191 clustering by extracted lncRNA profile showed distinct clinical relevance. Average tumor 192 diameter in G1 or G2 subtype were small size $(10.45 \pm 7.90 \mathrm{~mm})$ and were referred as benign or 193 early stage the GIST. G3 or G4 subtype showed larger size $(52.17 \pm 30.89 \mathrm{~mm})$, and were 
194

195

196

197

198

199

200

201

202

203

204

205

206

207

208

209

210

211

212

213

214

215

216

217

218

219

220

221

222

223

224

referred as malignant GIST tumor. Fisher's exact test showed that there were significant differences between class G1 and class G2 in the frequency of gender, as well as between subtypes G1/G2 and subtypes G3/G4 in the frequency of tumor size. LncRNAs' expression profile did not correlate with c-kit and PDGFRA mutation status, as well as age and tumor location (Table 1).

Previous research showed that methylation pattern of GISTs arising from the stomach segregated apart from GISTs from the intestine (Huang et al. 2016). Unlike methylation pattern indicating that GIST tissue of origin has a strong imprint on the epigenetic profile of this disease (Huang et al. 2016), lncRNA expression profile doesn't have correlation to stomach or intestine origin. Instead, the gender has significant influence on clustering by lncRNA expression profile, especially in small size GIST. This may be due to that the same cajai cells originate from alimentary track no matter where the GISTs locate. And the high expression abundance of gender-related lncRNA, such as XIST, FTX and TTY among the limited lncRNA-probes may also give much weight coefficient for this gender-oriented clustering.

\section{Female GIST patients showed favorable survival time by SEER database.}

Female and male patient showed distinct lncRNA expression profile especially in early stage of GIST and rapidly lost the gender-oriented clustering during the tumor progress, which suggest gender may be prognosis relevant as well as tumor size. So, we further explored the SEER database to analyze the survival rate differences between male and female GIST patients.

7983 eligible GIST patients were enrolled during the 41-year study period. The baseline characteristics of GIST patients stratified by gender were summarized in table 2 . The median survival time was 100 months (95\% CI 94.16-105.84) in male and 130 months (95\% CI 121.38138.62) in female GIST patients, female patients had a better overall survival (OS) than male patients. ( $\mathrm{HR}=1.277, \mathrm{P}<0.0001$, Fig. $2 \mathrm{~A})$.

Furthermore, 2567 female and 2567 male patients were perfect matched pairs for better analyses, baseline clinicopathological characteristics after perfect propensity-score-matched (PSM) analyses were also showed in table 2, the differences between two groups were eliminated with regard to primary tumor site, age, therapy, marital status, race, grade, tumor size (all factors $P=1.0)$. After PSM, the median survival time of male patients was 106 months (95\% CI 98.90-113.10), while matched female patients were 162 months (95\% CI 143.26-180.74). 
225 Consistently, female patients had a significantly longer overall survival time than male patients 226 in GIST $(\mathrm{HR}=1.470, \mathrm{P}<0.0001$, Fig. $2 \mathrm{~B})$.

227

228

229

Identification of biological processes and signaling pathways distinguishing certain subtype by gene set enrichment analysis (GSEA)

230 Significant gene sets were visualized as interaction networks with Cytoscape and Enrichment 231 Map (Merico et al. 2010). For analysis of male and female expression differences, we chose 18 232 female vs 18 male matched samples with equal tumor size for Cytoscape and Gene Set 233 Enrichment Analysis. Compared to female patients, interferon signaling, cytokine signaling in 234 immune system, immune checkpoint (PD1, TCR, CTLA4) and circadian expression were 235 significantly up-regulated in subtype of male patients (Fig. 3A-D). However, pathways of 236 oxidative phosphorylation, TCA cycle and respiratory electron transport were up-regulated in 237 subtype of female patients compared to male patients (Fig. 3A, E-G).

Subtypes G1 and G2, G3 and G4 were combined for analysis, and referred as G1/2 (small

239 size) and G3/4 (large size) respectively. Compared to subtype G1/2, most signaling pathways showed up-regulated in subtype G3/4, including DNA repair, carbohydrates metabolism, protein metabolism and lysosome pathway which might be involved in carcinogenesis or development of GIST (Fig. 3H, L-N). A few signaling pathways such as olfactory signaling, olfactory transduction signaling and hedgehog pathway were up-regulated in subtype G1/2 compared to 244 G3/4 (Fig. 3I-K).

245 These analysis results suggested that different gender and lncRNAs subtypes of GISTs 246 might arise from distinct abnormalities of biological signaling pathways.

\section{IncRNA Markers selection and associated expression pattern between 4 subtypes}

32 featured lncRNAs were identified to be significantly different expression between subtype G1/2 and subtype G3/4 by the Comparative Marker Selection algorithm, and their expression style were plotted in Figure 4A. As lncRNAs' functions are closely associated with their transcript abundance (Du et al. 2013), six lncRNAs LRRC75A-AS1, HYMAI, MALAT1, NEAT1, XIST and FTX with high expression value were recruited for further study. progression subtype G3/G4 compared with subtype G1/G2, whereas LRRC75A-AS1 showed the opposite upregulation (Fig.4B). High expression of LRRC75A-AS1 was positively associated with tumor diameters and seemed to be oncogene in GIST. XIST was specifically expressed in 
257 female GIST patients and was dramatically down-regulated in G3/G4 subtypes, which suggested 258 XIST was significantly inhibited during tumor progression and it seemed to have a protective 259 effect in female GIST patients. The expression of HYMAI, NEAT1, XIST and FTX were 260 negatively correlated with tumor diameter and significantly down-regulated during GIST 261 progression (Table 3). Overall, XIST down-regulation may suggest the malignant transformation 262 for female GIST patients, while down-regulation of HYMAI, NEAT1 and FTX, or up-regulation 263 of LRRC75A-AS1 may suggest the malignant transformation for both female and male patient, 264 which may be the promising biomarkers irrespective of small intestinal or gastric origin.

265

266

267

Protein-coding genes co-expressed with LRRC75A-AS1 and functional prediction

268

To further investigate the potential pathways associated with LRRC75A-AS1, the co-expressed protein-coding genes were computed using Pearson correlation coefficients (PCC) by R package LINC. There are 500 genes correlating with LRRC75A-AS1 (coefficient $>0.5$ ). Gene ontology (GO) and Kyoto Encyclopedia of Genes and Genomes (KEGG) analysis showed that LRRC75AAS1 co-expressed protein coding genes were mainly enriched in cadherin binding, unfolded protein and cell adhesion molecule binding, FK506 binding, isomerase and NADH activity (Fig. 4B). Taken together, these findings indicated that LRRC75A-AS1 may contribute to tumor malignancy by interacting with protein coding genes that involved in the above biological

276 pathways.

278

\section{IncRNA expression and CNA aberration}

LRRC75A-AS1 as a novel candidate oncogene in chromosome region 17p11.2-p12 had been

280 reported in human osteosarcoma to have a significant association between copy number and expression level (Both et al. 2012; Both et al. 2016). Although it also acted as an oncogene in GIST, LRRC75A-AS1 expression level showed no significant association with copy number alteration in our data analysis. Chromosome region 17p11.2-p12 showed a hotspot site in GIST, where LRRC75A-AS1 was significantly upregulated during tumor progression. Meanwhile chromosome region 17p11.2-p12 also contained protein-coding genes, RAI1, MSI2 and SMCR8, which were in co-expressed network of lncRNA HYMAI, NEAT1 and FTX (Fig. 5). Thus, 287 chromosome region 17p11.2-p12 may contribute to the oncogenic procession in malignant GIST. 


\section{Discussion}

289 Molecular expression profiles including the aberrant expressions of lncRNAs (Chen et al. 2014;

290 Qi \& Du 2013), have provided more information to help identify the molecular subtypes, grades

291 and prognosis of malignancy of various tumors (Chen et al. 2014; Sadanandam et al. 2013), as

292 well as in GIST (Lee et al. 2016; Niinuma et al. 2012). In this study, we first used mRNA gene

293 expression profiles to classify the GIST samples and didn't show any clinical meaning. Instead,

294 by profiling lncRNA expression, the GIST samples could be well clustered into four molecular

295 subtypes with distinct biological processes and different phenotype in their clinical character.

296 Thus, similar to other studies (Hessels et al. 2003; Prensner \& Chinnaiyan 2011), IncRNAs may

297 have higher specificity than mRNAs, and be more suitable to serve as prognostic and/or

298 predictive markers for GIST. In our present study, tumor location (stomach or intestine origin),

299 tyrosine kinase mutation status and age didn't show any association with lncRNA expression

300 profile. The major finding in our present study was that lncRNA expression profile well

301 clustered the GIST samples into small size $(<5 \mathrm{~mm})$ and large size tumors $(>5 \mathrm{~mm})$ which is a

302 fundamental index for GIST malignancy.

303 Interestingly, the gender had strong influence on clustering by lncRNA expression profile in

304 small size tumor (G1/G2). High expression of gender-related lncRNA, such as XIST, FTX and

305 TTTY15 among the limited lncRNA-probes may attribute to this gender-oriented clustering. The

306 great weight coefficient given by these gender-related lncRNAs were obvious in small size

307 tumor $(\mathrm{G} 1 / \mathrm{G} 2<5 \mathrm{~cm})$, and was rapidly lost when the tumor became malignant $(\mathrm{G} 3 / \mathrm{G} 4,>5 \mathrm{~cm})$

308 and the expression of XIST and FTX were significant inhibited. This expression pattern of XIST

309 in GIST was totally different from other tumors in previous published studies (Ma et al. 2017)

310 and meta-analysis (Zhu et al. 2018). Elevated lncRNA XIST expression predicts poor OS, poor

311 disease free survival (DFS), larger tumor size, increased distant metastasis and advanced tumor

312 stage in esophageal squamous cell carcinoma, pancreatic cancer, colorectal cancer, gastric

313 cancer, nasopharyngeal carcinoma, non-small cell lung cancer (Zhu et al. 2018) and

314 hepatocellular carcinoma (Ma et al. 2017). Here we demonstrated that female GISTs had a good

315 OS and the gender-related lncRNAs XIST, FTX might play a protective role for GIST

316 aggression.

317 The SEER data with 7983 GIST patients showed the favorable survival rate for female

318 patients $(\mathrm{P}<0.0001)$. In 2012 , the lancet oncology published a pooled population-based cohorts

319 consisting of 920 patients with GIST indicated that male sex were independent adverse 
320 prognostic factors (Joensuu et al. 2012), as well as large tumor size, high mitosis count, non-

321

322

323

324

325

326

327

328

329

330

331

332

333

334

335

336

337

338

339

340

341

342

343

344

345

346

347

348

349

350

351 gastric location, presence of rupture. Here we further investigated the molecular mechanism underlay clinical differences between gender. GSEA results showed in male patient, interferon signaling, cytokine signaling in immune system, immune checkpoint and circadian expression was significantly up-regulated while tricarboxylic acid cycle (TCA) cycle and respiratory electron transport pathways were down-regulated. Immune microenvironment profiling of gastrointestinal stromal tumors (GIST) shows gene expression patterns associated to immune checkpoint inhibitors response (Pantaleo et al. 2019). Gene expression profiles (GEP) and immunohistochemistry (IHC) from $31 \mathrm{KIT} / \mathrm{PDGFRA-mutant} \mathrm{GIST} \mathrm{supported} \mathrm{the} \mathrm{presence} \mathrm{of}$ immune infiltrate, IFN-gamma-induced immune signature (EIIS) and the T-cell-inflamed signature (TIS), which suggests that GIST may benefit from immunotherapy along with tyrosine kinase inhibitors (Pantaleo et al. 2019). Abundant infiltrating immune cells were found in PDGFRA-mutant GISTs and PD-L1 expression was negatively associated with tumor size(Sun et al. 2020). GSEA between small tumor size (G1/G2) and large tumor size (G3/G4) also showed significant differences, and gene set involved in carcinogenesis were identified as following: DNA repair, carbohydrates metabolism, protein metabolism and lysosome pathway were upregulated in subtype G3/G4. A few signaling pathways such as olfactory signaling, olfactory transduction signaling and hedgehog pathway were up-regulated in subtype G1/2. The previous results from immunohistochemical, immunofluorescence and ultrastructural techniques (Iruzubieta et al. 2020) showed Hedgehog signaling pathway activation (a pathway related with tumoral features such as proliferation, migration or stemness) mediated by primary cilia (an antenna-like structure based on microtubules presented in GIST cells) would be fundamental in tumoral microenvironment control of GIST cells for their maintenance, differentiation and proliferation. Hedgehog signaling could also activate KIT expression irrespective of mutation status, offering a novel approach to treat imatinib-resistant GIST (Tang et al. 2016). We also identified five lncRNAs with abundant expression (LRRC75A-AS1, HYMAI, NEAT1, XIST and FTX) were closely associated with tumor progress, which may suggest to be the biomarker for the malignancy of GIST. Particularly, LRRC75A-AS1 was positively corelated with tumor diameters and maybe an oncogene in GIST. Most recent study showed that Long non-coding RNA LRRC75A-AS1 facilitates triple negative breast cancer (TNBC) cell proliferation and invasion via functioning as a ceRNA network of LRRC75A-AS1/miR-380-3p/BAALC in accelerating TNBC development, indicating new promising targets for TNBC treatment(Li et al. 
352 353

354 355 356 357 358 359 360 361 362 363

364 365 366 367 368

369

370

371

372

373 374

375

376

377

378

379

380

381

382 383

2020). Previous studies reported lncRNAs HOTAIR(Lee et al. 2016; Niinuma et al. 2012), H19(Badalamenti et al. 2019) and $\mathrm{AOC} 4 \mathrm{P}(\mathrm{Hu}$ et al. 2018) are associated with the onset and progression of gastric GISTs and drive malignant character in GIST. In present study, the expression HOTAIR from the chip-derived data was very low, and both HOTAIR and H19 didn't shown any association with GIST clinicopathological value. AOC4P wasn't covered by this chip platform of Affymetrix HG-U133 Plus 2.0 arrays, analysis can't be performed.

Coincidentally, most of the featured lncRNAs are referred as chromatin associated RNAs (caRNAs) (Sridhar et al. 2017), including previously known XIST, NEAT1, MALAT1 (West et al. 2014). The chromatin associated long ncRNAs exert regulatory control on gene function through interaction with chromatin-associated proteins (Sridhar et al. 2017; West et al. 2014). So, modulation of chromatin structure maybe a mechanism of GIST carcinogenesis and aggression. We identified 9 protein-coding genes, which were significantly co-expressed with these featured lncRNAs and also differentially expressed between tumor stage. One of these protein genes, MSI2 was well defined as the loci target of NEAT1 previously (Sridhar et al. 2017; West et al. 2014). Oncogene-like LRRC75A-AS1, protein-coding co-expressed with caRNAs genes RAI1, SMCR8, and previous known NEAT1 targeted MSI2, were all at 17p11.2p12. Thus, chromosome region 17p11.2-p12 may contribute to the oncogenic procession in malignant GIST, which was similar to previous published study in human osteosarcoma (Both et al. 2012; Both et al. 2016). Circos plot showed that LRRC75A-AS1 had no significant association between copy number and gene expression level at site of 17p11.2-p12. Thus, expression activation of LRRC75A-AS1 in GIST was not similar as mechanism of concerted amplification-mediated overexpression with copy number aberration in human osteosarcoma (Both et al. 2012).

We also acknowledged some limitations of this study. First, the main drawback of present study is the lack of information on mitotic rate, which is one of two consensus factors for estimating the relative risk of GISTs (Fletcher et al. 2002). And the information of survival and clinical stage isn't available either. Second, Affymetrix HG-U133 Plus 2.0 arrays didn't cover all the possible lncRNAs present, the lncRNAs candidates identified here may not represent the complete lncRNA profiles underlying GIST clinical progression (Chen et al. 2014). To accurately and comprehensively elucidate the role of lncRNAs in classification of GIST, more comprehensive profiling studies and laboratory and clinical researches are needed. 


\section{Conclusions}

385 In summary, we identified four molecular subtypes in GIST based on the lncRNA profiles.

386 LncRNA expression between female and male showed distinct lncRNA profiles gene-set

387 pathways, and female GIST patients also showed favorable prognosis. LRRC75A-AS1 (Gene

388 ID: 125144, LRRC75A antisense RNA 1) may present an oncogene during the GIST

389 carcinogenesis. Modulation of chromatin structure by caRNAs, such as XIST, NEAT1 and

390 MALAT, maybe a mechanism of GIST carcinogenesis and aggression. Although the possible

391 functions of many identified lncRNA genes need to be further investigated, our study of the

392 lncRNA based classification may provide an efficient classification tool for marker selection and

clinical progression evaluation of GIST.

\section{References}

396

397

398

399

400

401

402

403

404

405

406

407

408

409

410

411

412

413

414

415

416

417

418

419

420

421

422

423

424

425

426

427

428

429

430

Astolfi A, Nannini M, Pantaleo MA, Di Battista M, Heinrich MC, Santini D, Catena F, Corless CL, Maleddu A, Saponara M, Lolli C, Di Scioscio V, Formica S, and Biasco G. 2010. A molecular portrait of gastrointestinal stromal tumors: an integrative analysis of gene expression profiling and high-resolution genomic copy number. Lab Invest 90:1285-1294. 10.1038/labinvest.2010.110

Badalamenti G, Barraco N, Incorvaia L, Galvano A, Fanale D, Cabibi D, Calo V, Curro G, Bazan V, and Russo A. 2019. Are Long Noncoding RNAs New Potential Biomarkers in Gastrointestinal Stromal Tumors (GISTs)? The Role of H19 and MALAT1. J Oncol 2019:5458717. 10.1155/2019/5458717

Both J, Wu T, Bras J, Schaap GR, Baas F, and Hulsebos TJ. 2012. Identification of novel candidate oncogenes in chromosome region 17p11.2-p12 in human osteosarcoma. PLoS One 7:e30907. 10.1371/journal.pone.0030907

Both J, Wu T, Ten Asbroek AL, Baas F, and Hulsebos TJ. 2016. Oncogenic Properties of Candidate Oncogenes in Chromosome Region 17p11.2p12 in Human Osteosarcoma. Cytogenet Genome Res 150:52-59. $10.1159 / 000451046$

Brunet JP, Tamayo P, Golub TR, and Mesirov JP. 2004. Metagenes and molecular pattern discovery using matrix factorization. Proc Natl Acad Sci U S A 101:4164-4169. 10.1073/pnas.0308531101

Chen H, Xu J, Hong J, Tang R, Zhang X, and Fang JY. 2014. Long noncoding RNA profiles identify five distinct molecular subtypes of colorectal cancer with clinical relevance. Mol Oncol 8:1393-1403. 10.1016/j.molonc.2014.05.010

Corless CL, Fletcher JA, and Heinrich MC. 2004. Biology of gastrointestinal stromal tumors. J Clin Oncol 22:38133825. 10.1200/JCO.2004.05.140

Cronin KA, Ries LA, and Edwards BK. 2014. The Surveillance, Epidemiology, and End Results (SEER) Program of the National Cancer Institute. Cancer 120 Suppl 23:3755-3757. 10.1002/cncr.29049

DeMatteo RP, Lewis JJ, Leung D, Mudan SS, Woodruff JM, and Brennan MF. 2000. Two hundred gastrointestinal stromal tumors: recurrence patterns and prognostic factors for survival. Ann Surg 231:51-58.

Du Z, Fei T, Verhaak RG, Su Z, Zhang Y, Brown M, Chen Y, and Liu XS. 2013. Integrative genomic analyses reveal clinically relevant long noncoding RNAs in human cancer. Nat Struct Mol Biol 20:908-913. $10.1038 / \mathrm{nsmb} .2591$

Fletcher CD, Berman JJ, Corless C, Gorstein F, Lasota J, Longley BJ, Miettinen M, O'Leary TJ, Remotti H, Rubin BP, Shmookler B, Sobin LH, and Weiss SW. 2002. Diagnosis of gastrointestinal stromal tumors: A consensus approach. Hum Pathol 33:459-465.

Gillis J, and Pavlidis P. 2012. "Guilt by association" is the exception rather than the rule in gene networks. PLoS Comput Biol 8:e1002444. 10.1371/journal.pcbi.1002444

Gould J, Getz G, Monti S, Reich M, and Mesirov JP. 2006. Comparative gene marker selection suite. Bioinformatics 22:1924-1925. 10.1093/bioinformatics/btl196

Hessels D, Klein Gunnewiek JM, van Oort I, Karthaus HF, van Leenders GJ, van Balken B, Kiemeney LA, Witjes 
431

432

433

434

435

436

437

438

439

440

441

442

443

444

445

446

447

448

449

450

451

452

453

454

455

456

457

458

459

460

461

462

463

464

465

466

467

468

469

470

471

472

473

474

475

476

477

478

479

480

481

482

483

484

485

486
JA, and Schalken JA. 2003. DD3(PCA3)-based molecular urine analysis for the diagnosis of prostate cancer. Eur Urol 44:8-15; discussion 15-16.

Hirota S, Isozaki K, Moriyama Y, Hashimoto K, Nishida T, Ishiguro S, Kawano K, Hanada M, Kurata A, Takeda M, Muhammad Tunio G, Matsuzawa Y, Kanakura Y, Shinomura Y, and Kitamura Y. 1998. Gain-of-function mutations of c-kit in human gastrointestinal stromal tumors. Science 279:577-580.

Hirota S, Ohashi A, Nishida T, Isozaki K, Kinoshita K, Shinomura Y, and Kitamura Y. 2003. Gain-of-function mutations of platelet-derived growth factor receptor alpha gene in gastrointestinal stromal tumors. Gastroenterology 125:660-667.

Hu JC, Wang Q, Jiang LX, Cai L, Zhai HY, Yao ZW, Zhang ML, and Feng Y. 2018. Effect of long non-coding RNA AOC4P on gastrointestinal stromal tumor cells. Onco Targets Ther 11:6259-6269. 10.2147/OTT.S174524

Hu Y, Chen HY, Yu CY, Xu J, Wang JL, Qian J, Zhang X, and Fang JY. 2014. A long non-coding RNA signature to improve prognosis prediction of colorectal cancer. Oncotarget 5:2230-2242. 10.18632/oncotarget.1895

Huang KK, McPherson JR, Tay ST, Das K, Tan IB, Ng CC, Chia NY, Zhang SL, Myint SS, Hu L, Rajasegaran V, Huang D, Loh JL, Gan A, Sairi AN, Sam XX, Dominguez LT, Lee M, Soo KC, Ooi LL, Ong HS, Chung A, Chow PK, Wong WK, Selvarajan S, Ong CK, Lim KH, Nandi T, Rozen S, Teh BT, Quek R, and Tan P. 2016. SETD2 histone modifier loss in aggressive GI stromal tumours. Gut 65:1960-1972. 10.1136/gutjnl2015-309482

Irizarry RA, Bolstad BM, Collin F, Cope LM, Hobbs B, and Speed TP. 2003a. Summaries of Affymetrix GeneChip probe level data. Nucleic Acids Res 31:e15.

Irizarry RA, Hobbs B, Collin F, Beazer-Barclay YD, Antonellis KJ, Scherf U, and Speed TP. 2003b. Exploration, normalization, and summaries of high density oligonucleotide array probe level data. Biostatistics 4:249-264. 10.1093/biostatistics/4.2.249

Iruzubieta P, Monzón M, Castiella T, Ramírez T, and Junquera C. 2020. Hedgehog signalling pathway activation in gastrointestinal stromal tumours is mediated by primary cilia. Gastric Cancer 23:64-72. 10.1007/s10120019-00984-2

Janeway KA, Kim SY, Lodish M, Nose V, Rustin P, Gaal J, Dahia PL, Liegl B, Ball ER, Raygada M, Lai AH, Kelly L, Hornick JL, Pediatric NIH, Wild-Type GC, O'Sullivan M, de Krijger RR, Dinjens WN, Demetri GD, Antonescu CR, Fletcher JA, Helman L, and Stratakis CA. 2011. Defects in succinate dehydrogenase in gastrointestinal stromal tumors lacking KIT and PDGFRA mutations. Proc Natl Acad Sci U S A 108:314318. 10.1073/pnas.1009199108

Joensuu H, Vehtari A, Riihimaki J, Nishida T, Steigen SE, Brabec P, Plank L, Nilsson B, Cirilli C, Braconi C, Bordoni A, Magnusson MK, Linke Z, Sufliarsky J, Federico M, Jonasson JG, Dei Tos AP, and Rutkowski P. 2012. Risk of recurrence of gastrointestinal stromal tumour after surgery: an analysis of pooled population-based cohorts. Lancet Oncol 13:265-274. 10.1016/S1470-2045(11)70299-6

Killian JK, Miettinen M, Walker RL, Wang Y, Zhu YJ, Waterfall JJ, Noyes N, Retnakumar P, Yang Z, Smith WI, Jr., Killian MS, Lau CC, Pineda M, Walling J, Stevenson H, Smith C, Wang Z, Lasota J, Kim SY, Boikos SA, Helman LJ, and Meltzer PS. 2014. Recurrent epimutation of SDHC in gastrointestinal stromal tumors. Sci Transl Med 6:268ra177. 10.1126/scitranslmed.3009961

Kindblom LG, Remotti HE, Aldenborg F, and Meis-Kindblom JM. 1998. Gastrointestinal pacemaker cell tumor (GIPACT): gastrointestinal stromal tumors show phenotypic characteristics of the interstitial cells of Cajal. Am J Pathol 152:1259-1269.

Lee NK, Lee JH, Kim WK, Yun S, Youn YH, Park CH, Choi YY, Kim H, and Lee SK. 2016. Promoter methylation of PCDH10 by HOTAIR regulates the progression of gastrointestinal stromal tumors. Oncotarget 7:7530775318. 10.18632/oncotarget.12171

Li S, Wu D, Jia H, and Zhang Z. 2020. Long non-coding RNA LRRC75A-AS1 facilitates triple negative breast cancer cell proliferation and invasion via functioning as a ceRNA to modulate BAALC. Cell Death Dis 11:643. 10.1038/s41419-020-02821-2

Lin R, Maeda S, Liu C, Karin M, and Edgington TS. 2007. A large noncoding RNA is a marker for murine hepatocellular carcinomas and a spectrum of human carcinomas. Oncogene 26:851-858. 10.1038/sj.onc. 1209846

Ma X, Yuan T, Yang C, Wang Z, Zang Y, Wu L, and Zhuang L. 2017. X-inactive-specific transcript of peripheral blood cells is regulated by exosomal Jpx and acts as a biomarker for female patients with hepatocellular carcinoma. Ther Adv Med Oncol 9:665-677. 10.1177/1758834017731052

Merico D, Isserlin R, Stueker O, Emili A, and Bader GD. 2010. Enrichment map: a network-based method for geneset enrichment visualization and interpretation. PLoS One 5:e13984. 10.1371/journal.pone.0013984

Mermel CH, Schumacher SE, Hill B, Meyerson ML, Beroukhim R, and Getz G. 2011. GISTIC2.0 facilitates sensitive 
and confident localization of the targets of focal somatic copy-number alteration in human cancers. Genome Biol 12:R41. 10.1186/gb-2011-12-4-r41

Miettinen M, El-Rifai W, L HLS, and Lasota J. 2002. Evaluation of malignancy and prognosis of gastrointestinal stromal tumors: a review. Hum Pathol 33:478-483.

Miettinen M, and Lasota J. 2001. Gastrointestinal stromal tumors--definition, clinical, histological, immunohistochemical, and molecular genetic features and differential diagnosis. Virchows Arch 438:1-12.

Miettinen M, Wang ZF, Sarlomo-Rikala M, Osuch C, Rutkowski P, and Lasota J. 2011. Succinate dehydrogenasedeficient GISTs: a clinicopathologic, immunohistochemical, and molecular genetic study of 66 gastric GISTs with predilection to young age. Am J Surg Pathol 35:1712-1721. 10.1097/PAS.0b013e3182260752

Mitra SA, Mitra AP, and Triche TJ. 2012. A central role for long non-coding RNA in cancer. Front Genet 3:17. $10.3389 /$ fgene. 2012.00017

Niinuma T, Suzuki H, Nojima M, Nosho K, Yamamoto H, Takamaru H, Yamamoto E, Maruyama R, Nobuoka T, Miyazaki Y, Nishida T, Bamba T, Kanda T, Ajioka Y, Taguchi T, Okahara S, Takahashi H, Nishida Y, Hosokawa M, Hasegawa T, Tokino T, Hirata K, Imai K, Toyota M, and Shinomura Y. 2012. Upregulation of miR-196a and HOTAIR drive malignant character in gastrointestinal stromal tumors. Cancer Res 72:11261136. 10.1158/0008-5472.CAN-11-1803

Ostrowski J, Polkowski M, Paziewska A, Skrzypczak M, Goryca K, Rubel T, Kokoszynska K, Rutkowski P, Nowecki ZI, Vel Dobosz AJ, Jarosz D, Ruka W, and Wyrwicz LS. 2009. Functional features of gene expression profiles differentiating gastrointestinal stromal tumours according to KIT mutations and expression. $B M C$ Cancer 9:413. 10.1186/1471-2407-9-413

Pantaleo MA, Tarantino G, Agostinelli C, Urbini M, Nannini M, Saponara M, Castelli C, Stacchiotti S, Fumagalli E, Gatto L, Santini D, De Leo A, Marafioti T, Akarca A, Sabattini E, Pession A, Ardizzoni A, Indio V, and Astolfi A. 2019. Immune microenvironment profiling of gastrointestinal stromal tumors (GIST) shows gene expression patterns associated to immune checkpoint inhibitors response. Oncoimmunology 8:e1617588. $10.1080 / 2162402 x .2019 .1617588$

Prensner JR, and Chinnaiyan AM. 2011. The emergence of lncRNAs in cancer biology. Cancer Discov 1:391-407. 10.1158/2159-8290.CD-11-0209

Qi P, and Du X. 2013. The long non-coding RNAs, a new cancer diagnostic and therapeutic gold mine. Mod Pathol 26:155-165. 10.1038/modpathol.2012.160

Sadanandam A, Lyssiotis CA, Homicsko K, Collisson EA, Gibb WJ, Wullschleger S, Ostos LC, Lannon WA, Grotzinger C, Del Rio M, Lhermitte B, Olshen AB, Wiedenmann B, Cantley LC, Gray JW, and Hanahan D. 2013. A colorectal cancer classification system that associates cellular phenotype and responses to therapy. Nat Med 19:619-625. 10.1038/nm.3175

Sridhar B, Rivas-Astroza M, Nguyen TC, Chen W, Yan Z, Cao X, Hebert L, and Zhong S. 2017. Systematic Mapping of RNA-Chromatin Interactions In Vivo. Curr Biol 27:602-609. 10.1016/j.cub.2017.01.011

Sun X, Sun J, Yuan W, Gao X, Fu M, Xue A, Li H, Shu P, Fang Y, Hou Y, Shen K, Sun Y, Qin J, and Qin X. 2020. Immune Cell Infiltration and the Expression of PD-1 and PD-L1 in Primary PDGFRA-Mutant Gastrointestinal Stromal Tumors. J Gastrointest Surg. 10.1007/s11605-020-04860-8

Tang CM, Lee TE, Syed SA, Burgoyne AM, Leonard SY, Gao F, Chan JC, Shi E, Chmielecki J, Morosini D, Wang K, Ross JS, Kendrick ML, Bardsley MR, Siena M, Mao J, Harismendy O, Ordog T, and Sicklick JK. 2016. Hedgehog pathway dysregulation contributes to the pathogenesis of human gastrointestinal stromal tumors via GLI-mediated activation of KIT expression. Oncotarget 7:78226-78241. 10.18632/oncotarget.12909

Tinzl M, Marberger M, Horvath S, and Chypre C. 2004. DD3PCA3 RNA analysis in urine--a new perspective for detecting prostate cancer. Eur Urol 46:182-186; discussion 187. 10.1016/j.eururo.2004.06.004

West JA, Davis CP, Sunwoo H, Simon MD, Sadreyev RI, Wang PI, Tolstorukov MY, and Kingston RE. 2014. The long noncoding RNAs NEAT1 and MALAT1 bind active chromatin sites. Mol Cell 55:791-802. 10.1016/j.molcel.2014.07.012

Xu Z, Huo X, Tang C, Ye H, Nandakumar V, Lou F, Zhang D, Jiang S, Sun H, Dong H, Zhang G, Liu Z, Dong Z, Guo B, Yan H, Yan C, Wang L, Su Z, Li Y, Gu D, Zhang X, Wu X, Wei X, Hong L, Zhang Y, Yang J, Gong Y, Tang C, Jones L, Huang XF, Chen SY, and Chen J. 2014. Frequent KIT mutations in human gastrointestinal stromal tumors. Sci Rep 4:5907. 10.1038/srep05907

Yamaguchi U, Nakayama R, Honda K, Ichikawa H, Hasegawa T, Shitashige M, Ono M, Shoji A, Sakuma T, Kuwabara H, Shimada Y, Sasako M, Shimoda T, Kawai A, Hirohashi S, and Yamada T. 2008. Distinct gene expression-defined classes of gastrointestinal stromal tumor. $J$ Clin Oncol 26:4100-4108. 10.1200/JCO.2007.14.2331

Yan J, Chen D, Chen X, Sun X, Dong Q, Du Z, and Wang T. 2019. Identification of imatinib-resistant long non- 
543

544

545

546

547

548

549

550

551

552

553

554

555

556

557

558 coding RNAs in gastrointestinal stromal tumors. Oncol Lett 17:2283-2295. 10.3892/ol.2018.9821

Yan L, Zou L, Zhao W, Wang Y, Liu B, Yao H, and Yu H. 2015. Clinicopathological significance of c-KIT mutation in gastrointestinal stromal tumors: a systematic review and meta-analysis. Sci Rep 5:13718. $10.1038 /$ srep 13718

Yoon JH, Kim J, and Gorospe M. 2015. Long noncoding RNA turnover. Biochimie 117:15-21. 10.1016/j.biochi.2015.03.001

Yu G, Wang LG, Yan GR, and He QY. 2015. DOSE: an R/Bioconductor package for disease ontology semantic and enrichment analysis. Bioinformatics 31:608-609. 10.1093/bioinformatics/btu684

Zhang X, Sun S, Pu JK, Tsang AC, Lee D, Man VO, Lui WM, Wong ST, and Leung GK. 2012. Long non-coding RNA expression profiles predict clinical phenotypes in glioma. Neurobiol Dis 48:1-8. 10.1016/j.nbd.2012.06.004

Zhu J, Kong F, Xing L, Jin Z, and Li Z. 2018. Prognostic and clinicopathological value of long noncoding RNA XIST in cancer. Clin Chim Acta 479:43-47. 10.1016/j.cca.2018.01.005

Zhu X, Tian X, Yu C, Shen C, Yan T, Hong J, Wang Z, Fang JY, and Chen H. 2016. A long non-coding RNA signature to improve prognosis prediction of gastric cancer. Mol Cancer 15:60. 10.1186/s12943-016-0544-0 


\section{Figure 1}

Figure 1 Classification of GISTs into four subtypes based on extracted IncRNA profiles.

(A-F) Heatmap showing consensus NMF clustering analysis of discovery dataset

GSE8167_RAW and GSE17743_RAW by extracted IncRNA probe sets. When K=4, cophenetic coefficient was the maximum. (G-H) Metagenes analysis of extracted IncRNAs ordered by $\mathrm{K}=4$ clustering subtypes, with annotations of each subtype $\mathrm{G} 1$ to $\mathrm{G} 4$, and (I) height plot of linkage tree for the discovery dataset listed on the head of 4 subtypes heatmap, with annotations of each subtype G1 to G4.

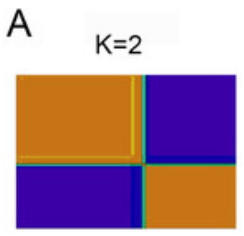
samples
Cophenetic coef. $=0.9915$ Cophenetic coef. $=0.9542$

D

$\mathrm{k}=5$

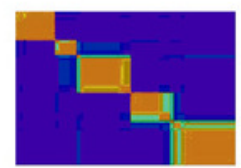

samples

$\begin{array}{ccc}\text { samples } & \text { samples } & \mathcal{U} \\ \text { Cophenetic } \text { coef. }=0.9908 \text { Cophenetic } \text { coef }=0.9625\end{array}$
B

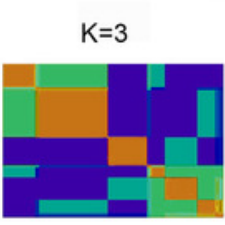

$\mathrm{E}$

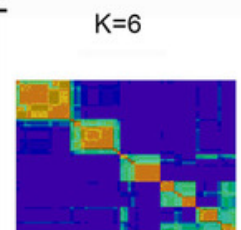

$\mathrm{F}$

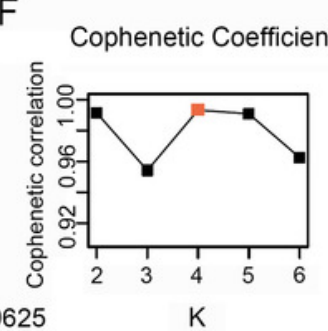

C

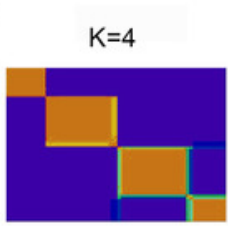
samples
Cophenetic coef. $=0.9932 \mathrm{H}$

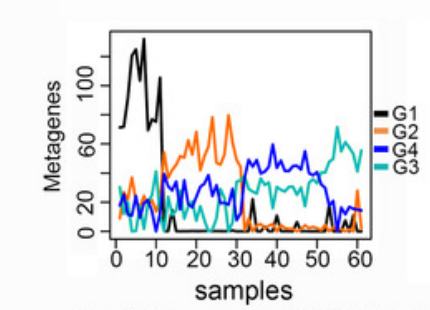
LncRNAprobeid_GIST61 K=4

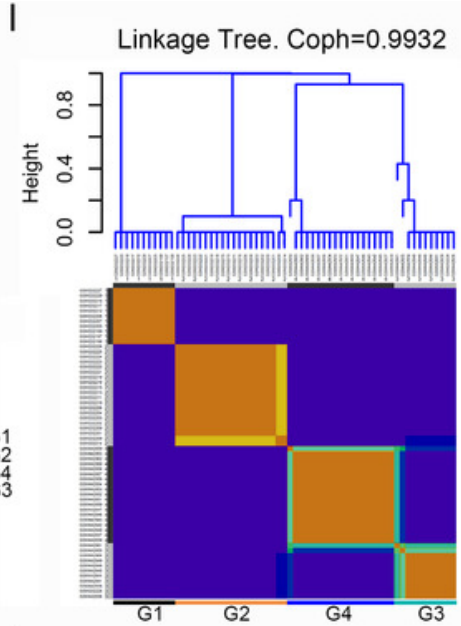


Figure 2

Figure 2 Cumulative survival curves of GIST patients stratified by gender from SEER database.

(A) Before and (B) after propensity score-match (PSM), Cumulative overall survival analysis of male and female GIST patients $(P<0.0001)$.

A

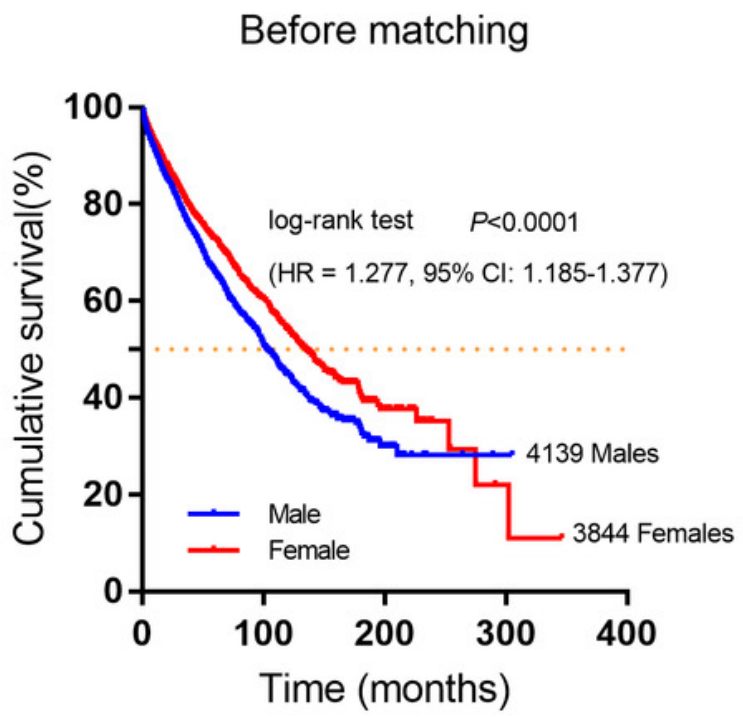

B

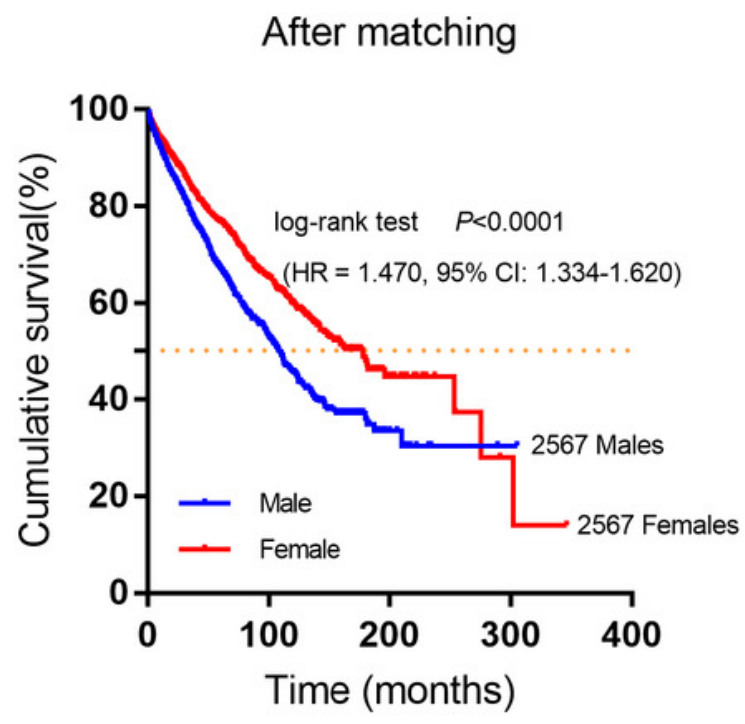




\section{Figure 3}

Figure 3 Gene set enrichment analyzes between subtypes by gender and subtypes by IncRNAs.

(A) Cytoscape map showing the upregulated and downregulated biological pathway in 18 male patients compared to matched 18 female GIST patients. (B-D) GSEA maps showing main upregulated pathway in male patients. (E-G) GSEA maps showing main upregulated pathway in female patients. (H) Cytoscape map showing the upregulated biological pathway in the subtype G3/4 GIST patients (tumor size $>5 \mathrm{~cm}$ ) compared to G1/2 GIST patients (tumor size $<5 \mathrm{~cm}$ ). (I-K) GSEA map showing the main upregulated pathway in G1/2 patients. (L-N) GSEA map showing the main upregulated pathway in G3/4 patients. Orange nodes mean upregulated gene sets, blue nodes mean downregulated gene sets, node size is proportional to the total number of genes within each gene set. 
A

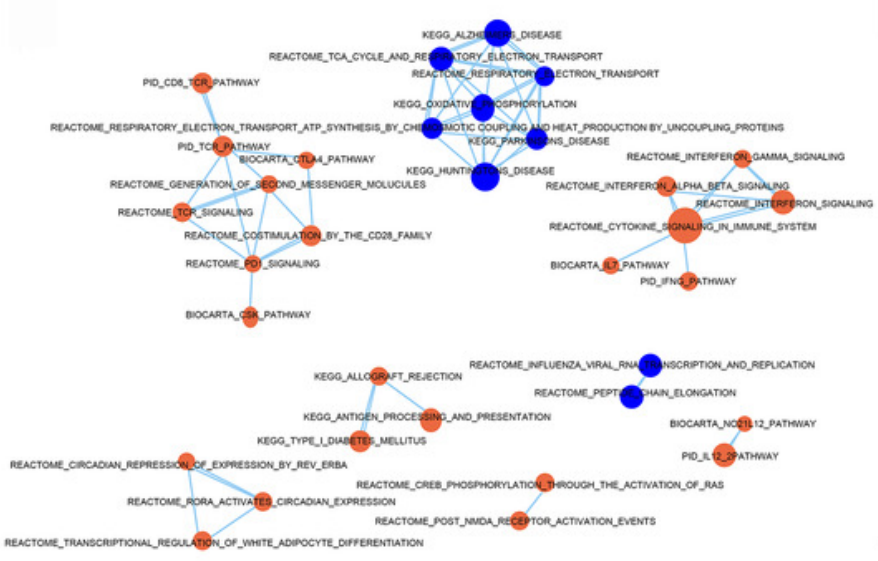

B

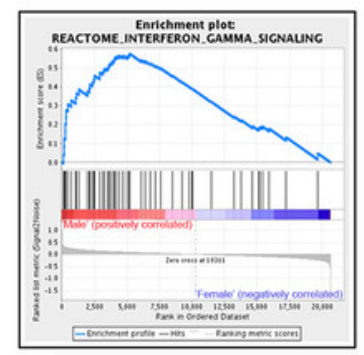

C

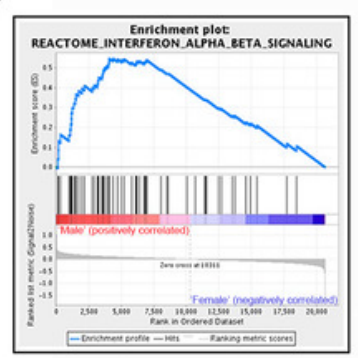

D

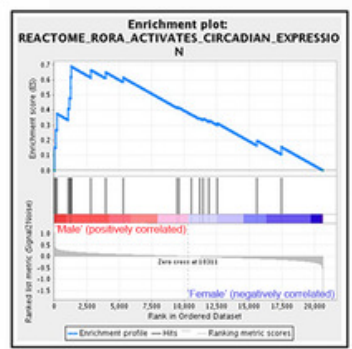

E

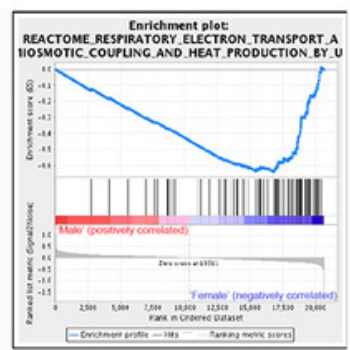

$\mathrm{F}$

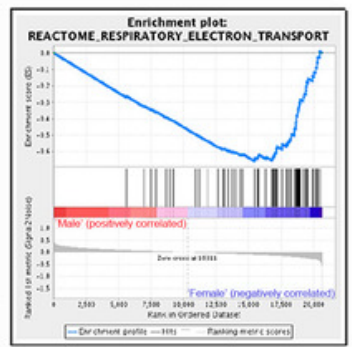

G

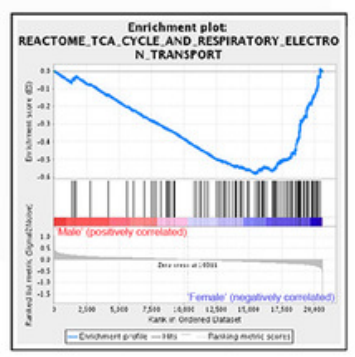

$\mathrm{H}$

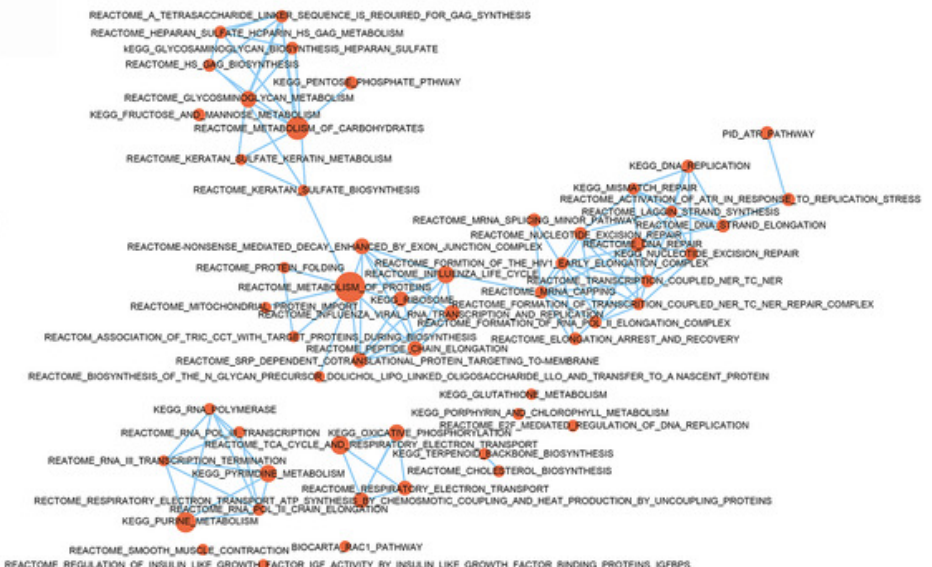

I

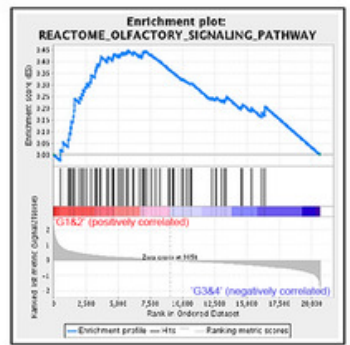

L

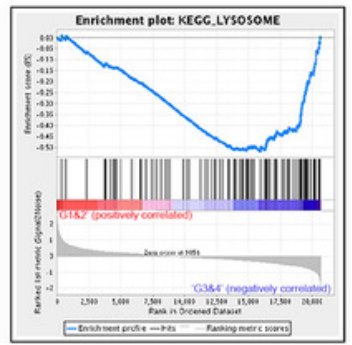

J

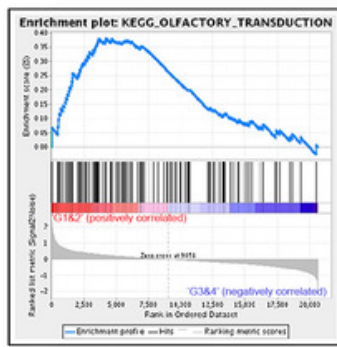

M

K

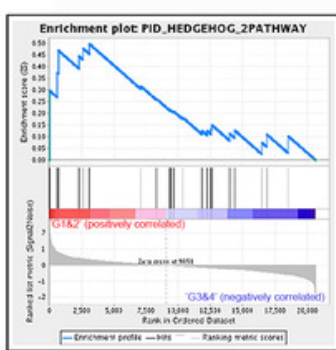

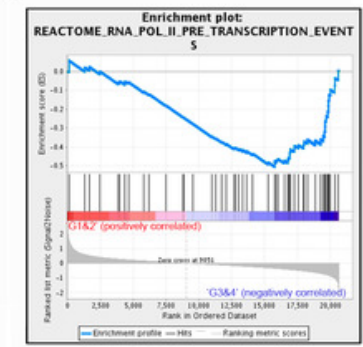

$\mathrm{N}$

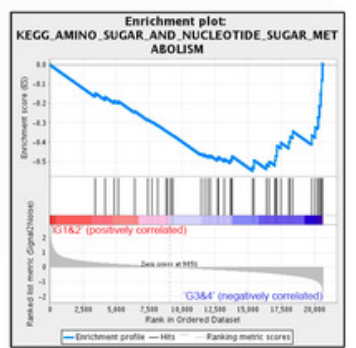


Figure 4

Figure 4 LncRNAs markers selection

(A) Comparative Marker Selection algorithm computed out 32 IncRNAs and their expression levels in 4 IncRNA clustering subtypes were plotted. (B) Functional enrichment analyses of the LRRC75A_AS1 co-expressed protein-coding genes by KEGG and GO analysis.

A

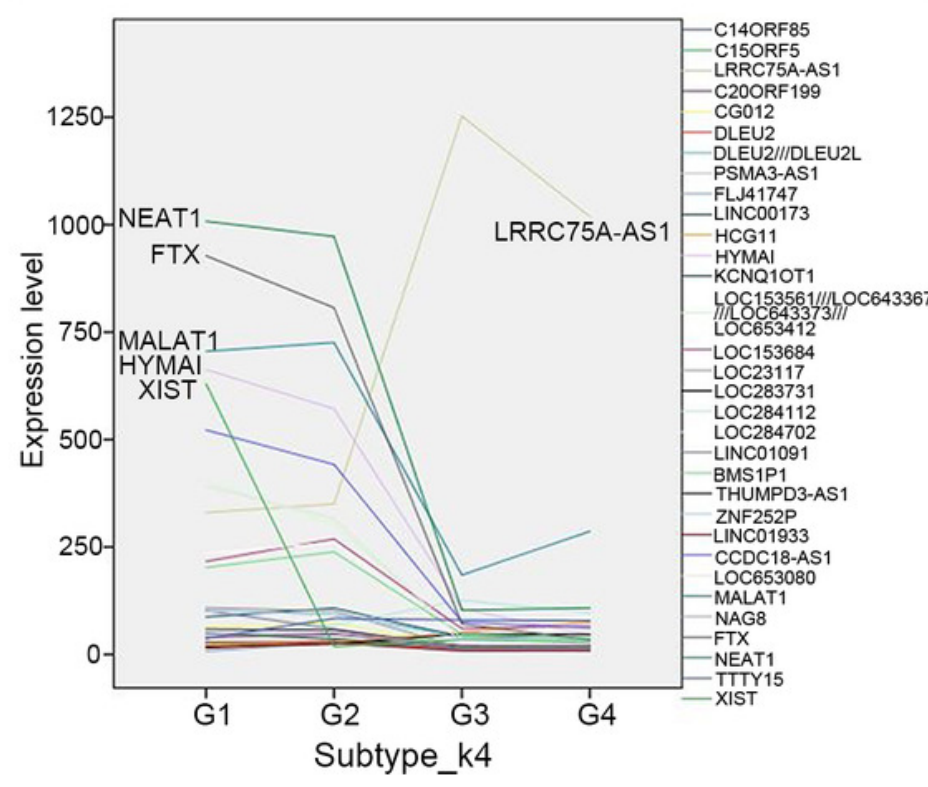

B

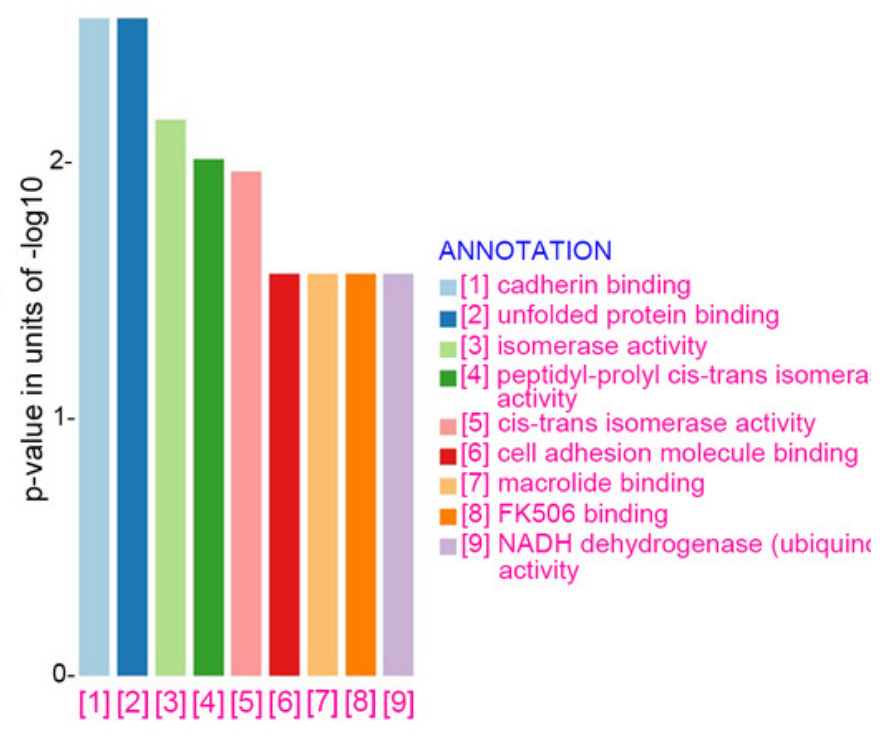




\section{Figure 5}

Figure 5 Integrated circos association map of signature IncRNAs, co-expressed proteincoding genes and copy number.

The outer ring shows ideogram of a normal karyotype. The next outermost red and blue labels index represents the most relevant IncRNAs markers. (Red represents IncRNA positive correlation with expression level changes and blue represents negative correlation). The inner ring represents copy number as a function of genomic coordinates. (Red represents amplification and green represents deletion). In the center of the figure, the connector showed the co-expressed protein-coding genes of representative abundant IncRNAs (LRRC75A-AS1, HYMAI, MALAT1, NEAT1, XIST and FTX $(p<0.001)$ ). 


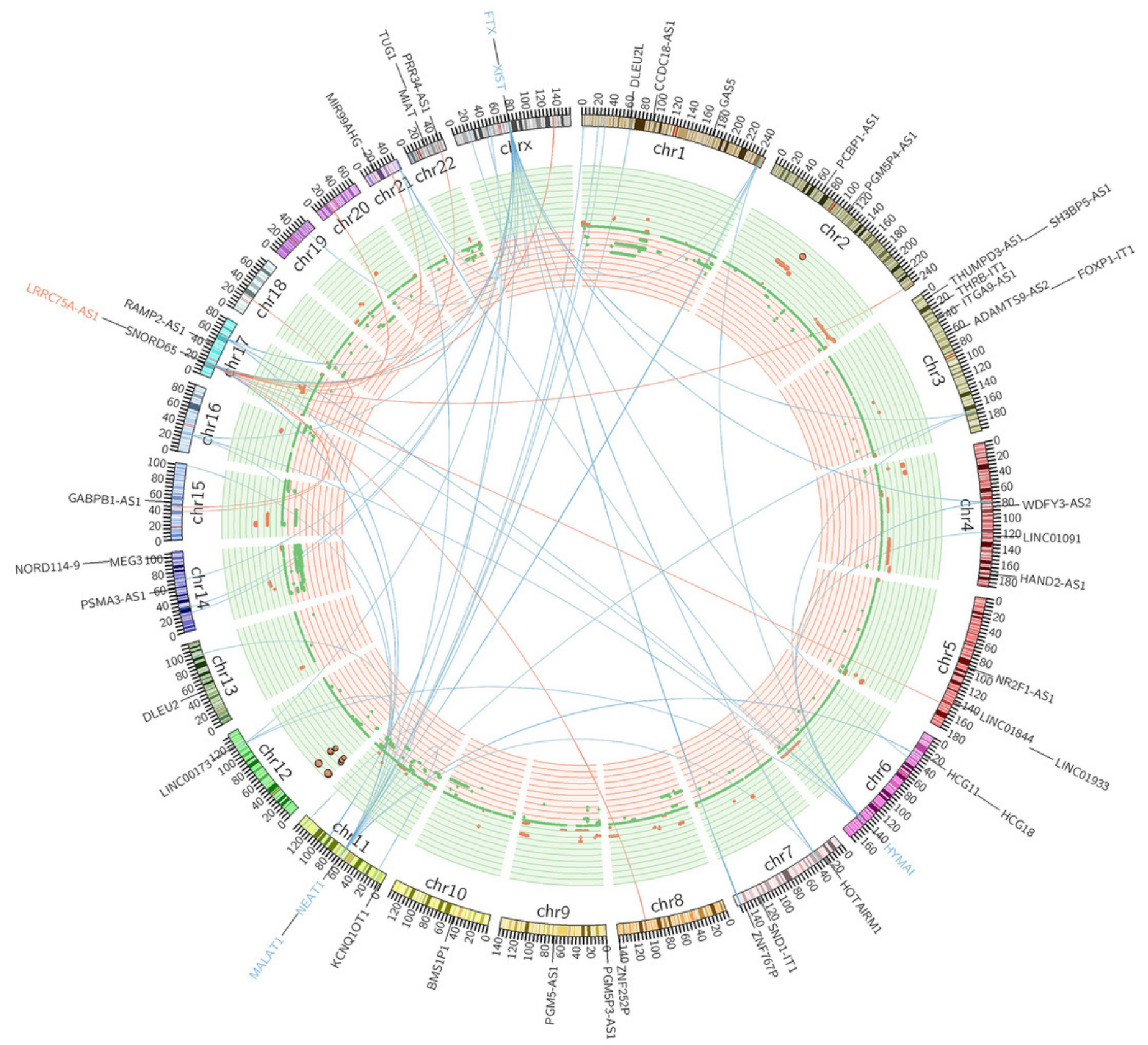




\section{Table $\mathbf{1}$ (on next page)}

Association analysis of clinical characteristics of GIST patients and 4 subtypes clustered by IncRNA profile

GIST: gastrointestinal stromal tumors; F:M: Female: Male; KIT: c-kit; WT: wild type; PDGRF: platelet-derived growth factor receptor alpha. ${ }^{a} \mathrm{p}$ values for diameter comparison between $\mathrm{G} 1$ and $\mathrm{G} 2$; ${ }^{b} \mathrm{p}$ values for diameter comparison between $\mathrm{G} 3$ and $\mathrm{G} 4{ }^{c} \mathrm{p}$ values for diameter comparison between $\mathrm{G} 1 / 2$ and $\mathrm{G} 3 / 4$. 
1 Table 1:

2 Association analysis of clinical characteristics of GIST patients and 4 subtypes clustered by IncRNA profile

\begin{tabular}{|c|c|c|c|c|c|c|c|c|c|}
\hline subtypes & cases & age & $\mathbf{F}: \mathbf{M}$ & $\begin{array}{c}\text { Location } \\
\text { Intestinal: } \\
\text { Gastric }\end{array}$ & $\begin{array}{l}\text { Mutation } \\
\text { KIT:WT: } \\
\text { PDGRF }\end{array}$ & $\begin{array}{l}\text { Diameter } \\
\text { (mm) }\end{array}$ & $\begin{array}{l}\text { Sig. } \\
\text { (p) }\end{array}$ & $\begin{array}{l}\text { Diameter } \\
(\mathbf{m m})\end{array}$ & $\begin{array}{l}\text { Sig. } \\
\text { (p) }\end{array}$ \\
\hline G1 & 11 & $59.0+10.8$ & $11: 0$ & $2: 9$ & $6: 5: 0$ & $9.09+7.34$ & \multirow{2}{*}{$0.995^{\mathrm{a}}$} & \multirow{2}{*}{$10.45+7.90 *$} & \multirow{4}{*}{$0.000^{\mathrm{c}}$} \\
\hline G2 & 20 & $63.6+12.4$ & $1: 19$ & $7: 13$ & $16: 4: 0$ & $11.2+8.28$ & & & \\
\hline G3 & 11 & - & $3: 8$ & $0: 11$ & $6: 1: 4$ & $48.73+25.16$ & \multirow{2}{*}{$0.921^{b}$} & \multirow{2}{*}{$52.17+30.89 *$} & \\
\hline G4 & 19 & - & $4: 15$ & $0: 19$ & $10: 2: 7$ & $54.16+34.27$ & & & \\
\hline
\end{tabular}

3 GIST: gastrointestinal stromal tumors; F:M: Female: Male; KIT: c-kit; WT: wild type; PDGRF: platelet-derived growth factor receptor alpha.

$4 \quad{ }^{a} \mathrm{p}$ values for diameter comparison between $\mathrm{G} 1$ and $\mathrm{G} 2$; ${ }^{\mathrm{b}} \mathrm{p}$ values for diameter comparison between $\mathrm{G} 3$ and $\mathrm{G} 4{ }^{\mathrm{c}} \mathrm{p}$ values for diameter comparison between $5 \mathrm{G} 1 / 2$ and $\mathrm{G} 3 / 4$. 


\section{Table 2 (on next page)}

Equilibrium comparison of baseline characteristics of GIST patients before and after propensity score matching. stratified by gender ( Male vs. Female). 
1 Table 2:

2 Equilibrium comparison of baseline characteristics of GIST patients before and after 3 propensity score matching. stratified by gender ( Male vs. Female).

\begin{tabular}{|c|c|c|c|c|c|c|}
\hline \multirow{3}{*}{ Characteristics } & \multicolumn{2}{|c|}{ Before matching } & \multicolumn{4}{|c|}{ After matching } \\
\hline & Male & Female & $\mathrm{p}$ & Male & Female & $\mathrm{p}$ \\
\hline & $4139(51.8 \%)$ & $3844(48.2 \%)$ & & $2567(50 \%)$ & $2567(50 \%)$ & \\
\hline Tumor site & & & 0.054 & & & 1.0 \\
\hline Stomach & $2192(53.0 \%)$ & $2188(56.9 \%)$ & & $1467(57.1 \%)$ & $1467(57.1 \%)$ & \\
\hline Small intestine & $1177(28.4 \%)$ & $977(25.4 \%)$ & & $716(27.9 \%)$ & $716(27.9 \%)$ & \\
\hline Rectum & $138(3.3 \%)$ & $85(2.2 \%)$ & & $37(1.4 \%)$ & $37(1.4 \%)$ & \\
\hline Colon & $112(2.7 \%)$ & $107(2.8 \%)$ & & $40(1.6 \%)$ & $40(1.6 \%)$ & \\
\hline Others & $520(12.6 \%)$ & $487(12.7 \%)$ & & $307(12.0 \%)$ & $307(12.0 \%)$ & \\
\hline Age & & & $<0.001$ & & & 1.0 \\
\hline$\leq 60$ & $1958(47.3 \%)$ & $1607(41.8 \%)$ & & $1259(49.0 \%)$ & $1259(49.0 \%)$ & \\
\hline$>60$ & $2181(52.7 \%)$ & $2237(58.2 \%)$ & & $1308(51.0 \%)$ & $1308(51.0 \%)$ & \\
\hline Therapy & & & $<0.001$ & & & 1.0 \\
\hline Surgery & $3190(77.1 \%)$ & $3086(80.3 \%)$ & & $2090(81.4 \%)$ & $2090(81.4 \%)$ & \\
\hline No surgery & $911(22.0 \%)$ & $731(19.0 \%)$ & & $473(18.4 \%)$ & $473(18.4 \%)$ & \\
\hline Unknown & $38(0.9 \%)$ & $27(0.7 \%)$ & & $4(0.2 \%)$ & $4(0.2 \%)$ & \\
\hline Marital status & & & $<0.001$ & & & 1.0 \\
\hline Married & $2751(66.5 \%)$ & $1861(48.4 \%)$ & & $1675(65.3 \%)$ & $1675(65.3 \%)$ & \\
\hline Widowed & $189(4.6 \%)$ & $773(20.1 \%)$ & & $157(6.1 \%)$ & $157(6.1 \%)$ & \\
\hline Single/Unmarried & $678(16.4 \%)$ & $636(16.5 \%)$ & & $438(17.1 \%)$ & $438(17.1 \%)$ & \\
\hline Divorced/separated & $318(7.7 \%)$ & $386(10.0 \%)$ & & $199(7.8 \%)$ & $199(7.8 \%)$ & \\
\hline Unknown & $203(4.9 \%)$ & $188(4.9 \%)$ & & $98(3.8 \%)$ & $98(3.8 \%)$ & \\
\hline Race & & & 0.62 & & & 1.0 \\
\hline White & $2891(69.8 \%)$ & $2578(67.1 \%)$ & & $1849(72.0 \%)$ & $1849(72.0 \%)$ & \\
\hline Black & $680(16.4 \%)$ & $729(19.0 \%)$ & & $399(15.5 \%)$ & $399(15.5 \%)$ & \\
\hline Others & $568(13.7 \%)$ & $537(14.0 \%)$ & & $319(12.4 \%)$ & $319(12.4 \%)$ & \\
\hline Grade & & & 0.37 & & & 1.0 \\
\hline $\mathrm{I} / \mathrm{II}$ & $715(17.3 \%)$ & $765(19.9 \%)$ & & $467(18.2 \%)$ & $467(18.2 \%)$ & \\
\hline III/IV & $482(11.6 \%)$ & $387(10.1 \%)$ & & $230(9.0 \%)$ & $230(9.0 \%)$ & \\
\hline Unknown & $2942(71.1 \%)$ & $2692(70.0 \%)$ & & $1870(72.8 \%)$ & $1870(72.8 \%)$ & \\
\hline
\end{tabular}




\begin{tabular}{ccccc} 
Tumor size & \multicolumn{5}{c}{$<0.001$} & & 1.0 \\
$\leq 2 \mathrm{~cm}$ & $203(4.9 \%)$ & $276(7.2 \%)$ & $121(4.7 \%)$ & $121(4.7 \%)$ \\
$2-5 \mathrm{~cm}$ & $717(17.3 \%)$ & $775(7.2 \%)$ & $480(18.7 \%)$ & $480(18.7 \%)$ \\
$5-10 \mathrm{~cm}$ & $933(22.5 \%)$ & $870(22.6 \%)$ & $578(22.5 \%)$ & $578(22.5 \%)$ \\
$>10 \mathrm{~cm}$ & $2286(55.2 \%)$ & $1923(50.0 \%)$ & $1388(54.1 \%)$ & $1388(54.1 \%)$ \\
\hline
\end{tabular}

4 


\section{Table 3(on next page)}

Co-relation analysis between featured IncRNA and clinical characteristics.

Coef.: Pearson correlation coefficient. $* P<0.05$ and $* * P<0.01$ 
1 Table 3:

2 Co-relation analysis between featured IncRNA and clinical characteristics.

\begin{tabular}{|cccccccc|}
\hline \multirow{2}{*}{$\begin{array}{c}\text { Pearson } \\
\text { correlation }\end{array}$} & & $\begin{array}{c}\text { LRRC75A } \\
\text {-AS1 }\end{array}$ & HYMAI & MALAT1 & NEAT1 & XIST & FTX \\
\hline \multirow{2}{*}{ Age } & Coef. & 0.059 & 0.163 & -0.314 & $-0.424^{*}$ & 0.179 & -0.244 \\
\cline { 2 - 7 } & $\mathrm{p}$ & 0.747 & 0.373 & 0.080 & 0.016 & 0.327 & 0.179 \\
\hline \multirow{2}{*}{ Dimeter } & Coef. & $0.610^{* *}$ & $-0.580^{* *}$ & 0.171 & $-0.415^{* *}$ & $-0.330^{* *}$ & $-0.439^{* *}$ \\
\cline { 2 - 7 } & $\mathrm{p}$ & 0.000 & 0.000 & 0.189 & 0.001 & 0.009 & 0.000 \\
\hline \multirow{2}{*}{ Group_k4 } & Coef. & $0.711^{* *}$ & $-0.680^{* *}$ & 0.196 & $-0.541^{* *}$ & $-0.646^{* *}$ & $-0.629^{* *}$ \\
& $\mathrm{p}$ & 0.000 & 0.000 & 0.130 & 0.000 & 0.000 & 0.000 \\
\hline
\end{tabular}

3 Coef.: Pearson correlation coefficient.

$4 \quad * P<0.05$ and $* * P<0.01$

5 